\title{
MIXING RANK-ONE ACTIONS FOR INFINITE SUMS OF FINITE GROUPS
}

\author{
Alexandre I. Danilenko
}

\begin{abstract}
Let $G$ be a countable direct sum of finite groups. We construct an uncountable family of pairwise disjoint mixing (of any order) rank-one strictly ergodic free actions of $G$ on a Cantor set. All of them possess the property of minimal selfjoinings (of any order). Moreover, an example of rigid weakly mixing rank-one strictly ergodic free $G$-action is given.
\end{abstract}

\section{INTRODUCTION AND DEFINITIONS}

This paper was inspired by the following question of D. Rudolph:

Question. Which countable discrete amenable groups $G$ have mixing (funny) rank one free actions?

Recall that a measure preserving action $T=\left(T_{g}\right)_{g \in G}$ of $G$ on a standard probability space $(X, \mathfrak{B}, \mu)$ is called

- mixing if $\lim _{g \rightarrow \infty} \mu\left(A \cap T_{g} B\right)=\mu(A) \mu(B)$ for all $A, B \in \mathfrak{B}$,

- mixing of order $l$ if for any $\epsilon>0$ and $A_{0}, \ldots, A_{l} \in \mathfrak{B}$, there exists a finite subset $K \subset G$ such that

$$
\left|\mu\left(T_{g_{0}} A_{0} \cap \cdots \cap T_{g_{l}} A_{l}\right)-\mu\left(A_{0}\right) \cdots \mu\left(A_{l}\right)\right|<\epsilon
$$

for each collection $g_{0}, \ldots, g_{l} \in G$ with $g_{i} g_{j}^{-1} \notin K$ if $i \neq j$,

- weakly mixing if the diagonal action $T \times T:=\left(T_{g} \times T_{g}\right)_{g \in G}$ of $G$ on the product space $(X \times X, \mathfrak{B} \otimes \mathfrak{B}, \mu \times \mu)$ is ergodic,

- totally ergodic if every co-finite subgroup in $G$ acts ergodically,

- rigid if there exists a sequence $g_{n} \rightarrow \infty$ in $G$ such that $\lim _{n \rightarrow \infty} \mu(A \cap$ $\left.T_{g_{n}} B\right) \rightarrow \mu(A \cap B)$ for all $A, B \in \mathfrak{B}$.

We say that $T$ has funny rank one if there exist a sequence of measurable subsets $\left(A_{n}\right)_{n=1}^{\infty}$ in $X$ and a sequence of finite subsets $\left(F_{n}\right)_{n=1}^{\infty}$ in $G$ such that the subsets $T_{g} F_{n}, g \in F_{n}$, are pairwise disjoint for any $n$ and

$$
\lim _{n \rightarrow \infty} \min _{H \subset F_{n}} \mu\left(B \triangle \bigsqcup_{g \in H} T_{g} A_{n}\right)=0 \text { for every } B \in \mathfrak{B} .
$$

1991 Mathematics Subject Classification. 37A40.

Key words and phrases. Joining, mixing, rank-one action.

The work was supported in part by CRDF, grant UM1-2546-KH-03. 
If, moreover, $\left(F_{n}\right)_{n=1}^{\infty}$ is a subsequence of some 'natural' Følner sequence in $G$, we say that $T$ has rank one. For instance, if $G=\mathbb{Z}^{d}$, this 'natural sequence' is just the sequence of cubes; if $G=\sum_{i=1}^{\infty} G_{i}$ with every $G_{i}$ a finite group, the sequence $\sum_{i=1}^{n} G_{i}$ is 'natural', etc.

Up to now various examples of mixing rank-one actions were constructed for

$-G=\mathbb{Z}$ in $[\mathrm{Or}],[\mathrm{Ru}],[\mathrm{Ad}],[\mathrm{CrS}]$, etc.,

$-G=\mathbb{Z}^{2}$ in $[\mathrm{AdS}]$

- $G=\mathbb{R}$ in $[\mathrm{Pr}],[\mathrm{Fa}]$,

$-G=\mathbb{R}^{d_{1}} \times \mathbb{Z}^{d_{2}}$ in $[\mathrm{DaS}]$.

We also mention two more constructions of rank-one actions for

- $G=\mathbb{Z} \oplus \bigoplus_{n=1}^{\infty} \mathbb{Z} / 2 \mathbb{Z}$ in $[\mathrm{Ju}]$, where it was claimed that the $\mathbb{Z}$-subaction is mixing but it was only shown that it is weakly mixing, and

- $G$ is a countable Abelian group with a subgroup $\mathbb{Z}^{d}$ such that the quotient $G / \mathbb{Z}^{d}$ is locally finite in [Ma], where it was proved that a $\mathbb{Z}$-subaction is mixing and it was asked whether the whole action is mixing.

Notice that in all of these examples $G$ is Abelian and has elements of infinite order. In contrast to that we provide a different class of groups for which the answer to the question of D. Rudolph is affirmative.

Theorem 0.1. Let $G=\bigoplus_{i=1}^{\infty} G_{i}$, where $G_{i}$ is a non-trivial finite group for every $i$.

(i) There exist uncountably many pairwise disjoint (and hence pairwise nonisomorphic) mixing rank-one strictly ergodic actions of $G$ on a Cantor set. Moreover, these actions are mixing of any order.

(ii) There exists a weakly mixing rigid (and hence non-mixing) rank-one strictly ergodic action of $G$ on a Cantor set.

Concerning (i), it is worth to note that any mixing rank-one $\mathbb{Z}$-action is mixing of any order by [Ka] and [Ry] (see also an extension of that to actions of some Abelian groups with elements of infinite order in $[\mathrm{JuY}]$ ). We do not know whether this fact holds for all mixing rank-one action of countable sums of finite groups.

To prove the theorem, we combine the original Ornstein's idea of 'random spacer' (in the cutting-and-stacking construction process) $[\mathrm{Or}]$ and the more recent $(C, F)$ construction developed in [Ju], [Da1], [Da2], [DaS1], [DaS2] to produce funny rankone actions with various dynamical properties. However, unlike all of the known examples of $(C, F)$-actions, the actions in this paper are constructed without adding any spacer (cf. with [Ju], where all the spacers relate to $\mathbb{Z}$-subaction only). Instead of that on the $n$-th step we just cut the $n$-'column' into 'subcolumns' and then rotate each 'subcolumn' in a 'random way'. In the limit we obtain a topological $G$-action on a compact Cantor space.

Our next concern is to describe all ergodic self-joinings of the $G$-actions constructed in Theorem 0.1. Recall a couple of definitions.

Given two ergodic $G$-actions $T$ and $T^{\prime}$ on $(X, \mathfrak{B}, \mu)$ and $\left(X^{\prime}, \mathfrak{B}^{\prime}, \mu^{\prime}\right)$ respectively, we denote by $J\left(T, T^{\prime}\right)$ the set of joinings of $T$ and $T^{\prime}$, i.e. the set of $\left(T_{g} \times T_{g}^{\prime}\right)_{g \in G^{-}}$ invariant measures on $\mathfrak{B} \otimes \mathfrak{B}^{\prime}$ whose marginals on $\mathfrak{B}$ and $\mathfrak{B}^{\prime}$ are $\mu$ and $\mu^{\prime}$ respectively. The corresponding dynamical system $\left(X \times X^{\prime}, \mathfrak{B} \otimes \mathfrak{B}^{\prime}, \mu \times \mu^{\prime}\right)$ is also called a joining of $T$ and $T^{\prime}$. By $J^{e}\left(T, T^{\prime}\right) \subset J(T, T)$ we denote the subset of ergodic joinings of $T$ and $T^{\prime}$ (it is never empty). In a similar way one can define the joininings $J\left(T_{1}, \ldots, T_{l}\right)$ for any finite family $T_{1}, \ldots, T_{l}$ of $G$-actions. If 
$J\left(T_{1}, \ldots, T_{l}\right)=\left\{\mu_{1} \times \cdots \times \mu_{l}\right\}$ then the family $T_{1}, \ldots, T_{l}$ is called disjoint. If $T_{1}=\cdots=T_{l}$ we speak about $l$-fold self-joinings of $T_{1}$ and use notation $J_{l}(T)$ for $J(\underbrace{T, \ldots, T}_{l \text { times }})$. For $g \in G$, we denote by $g^{\bullet}$ the conjugacy class of $g$. We also let

$$
\mathrm{FC}(G):=\left\{g \in G \mid g^{\bullet} \text { is finite }\right\} .
$$

Clearly, $\operatorname{FC}(G)$ is a normal subgroup of $G$. If $G$ is Abelian or $G$ is a sum of finite groups then $\mathrm{FC}(G)=G$. For any $g \in \mathrm{FC}(G)$, we define a measure $\mu_{g} \bullet$ on $(X \times X, \mathfrak{B} \otimes \mathfrak{B})$ by setting

$$
\mu_{g} \bullet(A \times B):=\frac{1}{\# g^{\bullet}} \sum_{h \in g} \mu\left(A \cap T_{h} B\right) .
$$

It is easy to verify that $\mu_{g} \bullet$ is a self-joining of $T$. Moreover, the map $\left(x, T_{h}^{-1} x\right) \mapsto$ $(x, h)$ is an isomorphism of $\left(X \times X, \mu_{g} \bullet, T \times T\right)$ onto $\left(X \times g^{\bullet}, \mu \times \nu, \widetilde{T}\right)$, where $\nu$ is the equidistribution on $g^{\bullet}$ and the $G$-action $\widetilde{T}=\left(\widetilde{T}_{t}\right)_{t \in G}$ is given by

$$
\widetilde{T}_{t}(x, h)=\left(T_{t} x, t h t^{-1}\right), x \in X, h \in g^{\bullet} .
$$

It follows that $\widetilde{T}$ (and hence the self-joining $\mu_{g} \bullet$ of $T$ ) is ergodic if and only if the action $\left(T_{t}\right)_{t \in C(g)}$ is ergodic, where $C(g)=\{t \in G \mid t g=g t\}$ stands for the centralizer of $g$ in $G$. Notice also that $C(g)$ is a co-finite subgroup of $G$ because of $g \in \mathrm{FC}(G)$. Hence $\left\{\mu_{g} \bullet \mid g \in \mathrm{FC}(G)\right\} \subset J_{2}^{e}(T)$ whenever $T$ is totally ergodic.

Definition 0.2. If $J_{2}^{e}(T) \subset\left\{\mu_{g} \bullet \mid g \in \mathrm{FC}(G)\right\} \cup\{\mu \times \mu\}$ then we say that $T$ has 2-fold minimal self-joinings $\left(\mathrm{MSJ}_{2}\right)$.

This definition extends naturally to higher order self-joinings as follows. Given $l \geq 1$ and $g \in G^{l+1}$, we denote by $g^{\bullet l}$ the orbit of $g$ under the $G$-action on $G^{l+1}$ by conjugations:

$$
h \cdot\left(g_{0}, \ldots, g_{l}\right):=\left(h g_{0} h^{-1}, \ldots, h g_{l} h^{-1}\right) .
$$

Let $P$ be a partition of $\{0, \ldots, l\}$. For an atom $p \in P$, we denote by $i_{p}$ the minimal element in $p$. We say that an element $g=\left(g_{0}, \ldots, g_{l}\right) \in \mathrm{FC}(G)^{l+1}$ is $P$-subordinated if $g_{i_{p}}=1_{G}$ for all $p \in P$. For any such $g$, we define a measure $\mu_{g} \bullet$ on $\left(X^{l+1}, \mathfrak{B}^{\otimes(l+1)}\right)$ by setting

$$
\mu_{g \bullet l}\left(A_{0} \times \cdots \times A_{l}\right):=\frac{1}{\# g^{\bullet l}} \sum_{\left(h_{0}, \ldots, h_{l}\right) \in g^{\bullet l}} \prod_{p \in P} \mu\left(\bigcap_{i \in p} T_{h_{i}} A_{i}\right) .
$$

It is easy to verify that $\mu_{g} \bullet$ is an $(l+1)$-fold self-joining of $T$. Reasoning as above one can check that $\mu_{g} \bullet$ is ergodic whenever $T$ is weakly mixing.

Definition 0.3. We say that $T$ has $(l+1)$-fold minimal self-joinings $\left(\mathrm{MSJ}_{l+1}\right)$ if

$$
J_{l+1}^{e}(T) \subset\left\{\mu_{g \bullet l} \mid g \text { is } P \text {-subordinated for a partition } P \text { of }\{0, \ldots, l\}\right\} .
$$

If $T$ has $\mathrm{MSJ}_{l}$ for any $l>1$, we say that $T$ has MSJ.

In case $G$ is Abelian, these definitions agree with the - common now-definitions of $\mathrm{MSJ}_{l+1}$ and MSJ by A. del Junco and D. Rudolph [JuR] who considered selfjoinings $\mu_{g} \bullet$ only when $g$ belongs to the center of $G^{l+1}$. However we find their definition somewhat restrictive for non-commutative groups since, for instance, countable sums of non-commutative finite groups can never have actions with $\mathrm{MSJ}_{2}$ in their sense.

Now we record the second main result of this paper. 
Theorem 0.4. The actions constructed in Theorem 0.1(i) all have MSJ.

We notice that a part of the analysis from $[\mathrm{Ru}]$ can be carried over to the case of $G$-actions with MSJ. In this paper we only show that such actions have trivial product centralizer. Moreover, as follows from [Da3], every $G$-action with $\mathrm{MSJ}_{2}$ is effectively prime, i.e. has no factors except for the obvious ones: the sub- $\sigma$-algebras of subsets fixed by finite normal subgroups in $G$. In particular, there exist no free factors.

We now briefly summarize the organization of the paper. In Section 1 we outline the $(C, F)$-construction of rank-one actions as it appeared in [Da1]. In Section 2, for any countable sum $G$ of finite groups, we construct a $(C, F)$-action $T$ of $G$ which is mixing of any order. A rigid weakly mixing action of $G$ also appears there. In Section 3 we demonstrate that $T$ has MSJ. In Section 4 we show how to perturb the construction of $T$ to obtain an uncountable family of pairwise disjoint mixing rank-one $G$-actions with MSJ. In the final Section 5 we discuss some implications of MSJ: trivial centralizer, trivial product centralizer and effective primality.

Acknowledgement. The author thanks the referee for the useful suggestions that improved the paper. In particular, in the present proof of Theorem 0.4 we deduce $\mathrm{MSJ}_{l}$ from the $l$-fold mixing (as J. King does for $\mathbb{Z}$-actions in $[\mathrm{Ki}]$ ). Our original proof (independent of multiple mixing) was longer and noticeably more complicated.

\section{1. $(C, F)$-CONSTRUCTION}

In this section we recall the $(C, F)$-construction of rank-one actions.

From now on $G=\sum_{i=1}^{\infty} G_{i}$, where $G_{i}$ is a non-trivial finite group for each $i \geq 1$. To construct a probability preserving $(C, F)$-action of $G$ (see [Ju], [Da1], [DaS2]) we need to define two sequences $\left(F_{n}\right)_{n \geq 0}$ and $\left(C_{n}\right)_{n \geq 1}$ of finite subsets in $G$ such that the following are satisfied:

$$
\left(F_{n}\right)_{n \geq 0} \text { is a Folner sequence in } G, F_{0}=\left\{1_{G}\right\} \text {, }
$$$$
F_{n} C_{n+1} \subset F_{n+1}, C_{n+1}>1 \text {, }
$$$$
F_{n} c \cap F_{n} c^{\prime}=\emptyset \text { for all } c \neq c^{\prime} \in C_{n+1} \text {, }
$$

$$
\lim _{n \rightarrow \infty} \frac{\# F_{n}}{\# C_{1} \cdots \# C_{n}}<\infty \text {. }
$$

Suppose that an increasing sequence of integers $0<k_{1}<k_{2}<\cdots$ is given. Then we define $\left(F_{n}\right)_{n \geq 0}$ by setting $F_{0}:=\left\{1_{G}\right\}$ and $F_{n}:=\sum_{i=1}^{k_{n}} G_{i}$ for $n \geq 1$. Clearly, (1-1) is satisfied. Suppose now that we are also given a sequence of maps $s_{n}: H_{n} \rightarrow F_{n}$, where $H_{0}:=\sum_{i=1}^{k_{1}} G_{i}$ and $H_{n}:=\sum_{i=k_{n}+1}^{k_{n+1}} G_{i}$ for $n \geq 1$. Then we define two sequences of maps $c_{n+1}, \phi_{n}: H_{n} \rightarrow F_{n+1}$ by setting $\phi_{n}(h):=(0, h)$ and $c_{n+1}(h):=\left(s_{n}(h), h\right)$. Finally, we let $C_{n+1}:=c_{n+1}\left(H_{n}\right)$ for all $n \geq 0$. It is easy to verify that (1-2)-(1-4) are all fulfilled. Moreover, a stronger version of (1-2) holds:

$$
F_{n} C_{n+1}=F_{n+1} \text {. }
$$

We now put $X_{n}:=F_{n} \times C_{n+1} \times C_{n+2} \times \cdots$ and define a map $i_{n}: X_{n} \rightarrow X_{n+1}$ by setting

$$
i_{n}\left(f_{n}, d_{n+1}, d_{n+2}, \ldots\right):=\left(f_{n} d_{n+1}, d_{n+2}, \ldots\right) .
$$

Clearly, $X_{n}$ is a compact Cantor space. It follows from (1-5) and (1-3) that $i_{n}$ is well defined and it is a homeomorphism of $X_{n}$ onto $X_{n+1}$. Denote by $X$ the 
topological inductive limit of the sequence $\left(X_{n}, i_{n}\right)_{n=1}^{\infty}$. As a topological space $X$ is canonically homeomorphic to any $X_{n}$ and in the sequel we will often identify $X$ with $X_{n}$ suppressing the canonical identification maps. We need the structure of inductive limit to define the $(C, F)$-action $T$ on $X$ as follows. Given $g \in G$, consider any $n \geq 0$ such that $g \in F_{n}$. Every $x \in X$ can be written as an infinite sequence $x=\left(f_{n}, d_{n+1}, d_{n+2}, \ldots\right)$ with $f_{n} \in F_{n}$ and $d_{m} \in C_{m}$ for $m>n$ (i.e. we identify $X$ with $\left.X_{n}\right)$. Now we put

$$
T_{g} x:=\left(g f_{n}, d_{n+1}, d_{n+2}, \ldots\right) \in X_{n} .
$$

It is easy to verify that $T_{g}$ is a well defined homeomorphism of $X$. Moreover, $T_{g} T_{g^{\prime}}=T_{g g^{\prime}}$, i.e. $T:=\left(T_{g}\right)_{g \in G}$ is a topological action of $G$ on $X$.

Definition 1.1. We call $T$ the $(C, F)$-action of $G$ associated with $\left(k_{n}, s_{n-1}\right)_{n=1}^{\infty}$.

We list without proof several properties of $T$. They can be verified easily by the reader (see also [Da1]).

- $T$ is a minimal uniquely ergodic (i.e. strictly ergodic) free action of $G$.

- Two points $x=\left(f_{n}, d_{n+1}, d_{n+2}, \ldots\right)$ and $x=\left(f_{n}^{\prime}, d_{n+1}^{\prime}, d_{n+2}^{\prime}, \ldots\right) \in X_{n}$ are $T$-orbit equivalent if and only if $d_{i}=d_{i}^{\prime}$ eventually (i.e. for all large enough $i$ ). Moreover, $x^{\prime}=T_{g} x$ if and only if

$$
g=\lim _{i \rightarrow \infty} f_{n}^{\prime} d_{n+1}^{\prime} \cdots d_{n+i}^{\prime} d_{n+i}^{-1} \cdots d_{n+1}^{-1} f_{n}^{-1} .
$$

- The only $T$-invariant probability measure $\mu$ on $X$ is the product of the equidistributions on $F_{n}$ and $C_{n+i}, i \in \mathbb{N}$ (if $X$ is identified with $X_{n}$ ).

For each $A \subset F_{n}$, we let $[A]_{n}:=\left\{x=\left(f_{n}, d_{n+1}, \ldots\right) \in X_{n} \mid f_{n} \in A\right\}$ and call it an $n$-cylinder. The following holds:

$$
\begin{aligned}
& {[A]_{n} \cap[B]_{n}=[A \cap B]_{n}, \text { and }[A]_{n} \cup[B]_{n}=[A \cup B]_{n},} \\
& {[A]_{n}=\bigsqcup_{d \in C_{n+1}}[A d]_{n+1},} \\
& T_{g}[A]_{n}=[g A]_{n} \text { if } g \in F_{n}, \\
& \mu\left([A d]_{n+1}\right)=\frac{1}{\# C_{n+1}} \mu\left([A]_{n}\right) \text { for any } d \in C_{n+1}, \\
& \mu\left([A]_{n}\right)=\lambda_{F_{n}}(A),
\end{aligned}
$$

where $\lambda_{F_{n}}$ is the normalized Haar measure on $F_{n}$. Moreover, for each measurable subset $B \subset X$,

$$
\lim _{n \rightarrow \infty} \min _{A \subset F_{n}} \mu\left(B \triangle[A]_{n}\right)=0 .
$$

Hence $T$ has rank one.

\section{Mixing $(C, F)$-ACTIONS}

Our purpose in this section is to construct a rank-one action of $G$ which is mixing of any order. This action will appear as a $(C, F)$-action associated with some specially selected sequence $\left(k_{n}, s_{n-1}\right)_{n \geq 1}$. We first state several preliminary results.

Given finite sets $A$ and $B$ and a map $x \in A^{B}$, we denote by dist $x$ or $\operatorname{dist}_{b \in B} x(b)$ the measure $(\# B)^{-1} \sum_{b \in B} \chi_{x(b)}$ on $A$. Here $\chi_{x(b)}$ stands for the probability supported at the point $x(b)$. 
Lemma 2.1. Let $A$ be a finite set and let $\lambda$ be the equidistribution on $A$. Then for any $\epsilon>0$ there exist $c>0$ and $m \in \mathbb{N}$ such that for any finite set $B$ with $\# B>m$,

$$
\lambda^{B}\left(\left\{x \in A^{B} \mid\|\operatorname{dist} x-\lambda\|>\epsilon\right\}\right)<e^{-c \# B} .
$$

For the proof we refer to [Or] or $[\mathrm{Ru}]$. We will also use the following combinatorial lemma.

Lemma 2.2. For any $l \in \mathbb{N}$, let $N_{l}:=3^{l(l-1) / 2}$ and $\delta_{l}:=5^{-l(l-1) / 2}$ Let $H$ be a finite group. Then for any family $h_{1}, \ldots, h_{l}$ of mutually different elements of $H$ and any subset $B \subset H$ with $\# B>3 / \delta_{l}$, there exists a partition of $B$ into subsets $B_{i}, 1 \leq i \leq N_{l}$, such that the subsets $h_{1} B_{i}, h_{2} B_{i}, \ldots, h_{l} B_{i}$ are mutually disjoint and $\# B_{i} \geq \delta_{l} \# B$ for any $i$.

Proof. We leave to the reader the simplest case when $l=2$. Hint: assume that $h_{1}=1_{H}$ and consider the partition of $H$ into the right cosets by the cyclic group generated by $h_{2}$.

Suppose that we already proved the assertion of the lemma for some $l$ and we want to prove it for $l+1$. Take any $h_{1} \neq h_{2} \neq \cdots \neq h_{l+1} \in H$ (in such a way we denote mutually different elements of $H$ ). Given a subset $B \subset H$ with $\# B>3 / \delta_{l}$, we first partition $B$ into subsets $B_{i}, 1 \leq i \leq N_{l}$, such that the subsets $h_{2} B_{i}, h_{3} B_{i}, \ldots, h_{l+1} B_{i}$ are mutually disjoint and $\# B_{i} \geq \delta_{l} \# B \geq 3 \cdot 5^{l}$. For every $i$, there exists a partition $B_{i}=\bigsqcup_{i_{1}=1}^{3} B_{i, i_{1}}$ such that $h_{1} B_{i, i_{1}} \cap h_{2} B_{i, i_{1}}=\emptyset$ and $\# B_{i, i_{1}} \geq 0.2 \# B_{i}, 1 \leq i_{1} \leq 3$. Next, we partition every $B_{i, i_{1}}$ into 3 subsets $B_{i, i_{1}, i_{2}}$ such that $h_{1} B_{i, i_{1}, i_{2}} \cap h_{3} B_{i, i_{1}, i_{2}}=\emptyset$ and $\# B_{i, i_{1}, i_{2}} \geq 0.2 \# B_{i, i_{1}}, 1 \leq i_{2} \leq 3$, and so on. Finally, we obtain a partition

$$
B=\bigsqcup_{i=1}^{N_{l}} \bigsqcup_{i_{1}, \ldots, i_{l}=1}^{3} B_{i, i_{1}, \ldots, i_{l}}
$$

which is as desired.

Given a finite set $A$, a finite group $H$ and elements $h_{1}, \ldots, h_{l} \in H$, we denote by $\pi_{h_{1}, \ldots, h_{l}}$ the map $A^{H} \rightarrow\left(A^{l}\right)^{H}$ given by

$$
\left(\pi_{h_{1}, \ldots, h_{l}} x\right)(k)=\left(x\left(h_{1} k\right), \ldots, x\left(h_{l} k\right)\right) .
$$

For $x \in A^{H}$, we define $x^{*} \in A^{H}$ by setting $x^{*}(h):=x\left(h^{-1}\right), h \in H$.

Lemma 2.3. Given $l \in \mathbb{N}$ and $\epsilon>0$, there exists $m \in \mathbb{N}$ such that for any finite group $H$ with $\# H>m$, one can find $s \in A^{H}$ such that

$$
\left\|\operatorname{dist} \pi_{h_{1}, \ldots, h_{l}} s-\lambda^{l}\right\|<\epsilon \text { and }\left\|\operatorname{dist} \pi_{h_{1}, \ldots, h_{l}} s^{*}-\lambda^{l}\right\|<\epsilon
$$

for all $h_{1} \neq h_{2} \neq \cdots \neq h_{l} \in H$.

Proof. Take any finite group $H$ and set

$$
B_{H}:=\bigcup_{h_{1} \neq \cdots \neq h_{l} \in H}\left\{x \in A^{H} \mid\left\|\operatorname{dist} \pi_{h_{1}, \ldots, h_{l}} x-\lambda^{l}\right\|>\epsilon\right\} .
$$


To prove the left hand side inequality in (2-1) it suffices to show that $\lambda^{H}\left(B_{H}\right)<1$ whenever $\# H$ is large enough. Moreover, since the map $A^{H} \ni x \mapsto x^{*} \in A^{H}$ preserves the measure $\lambda^{H}$, the right hand side inequality in (2-1) will follow from the left hand side one if we prove that $\lambda^{H}\left(B_{H}\right)<0.5$.

Fix $h_{1} \neq \cdots \neq h_{l} \in H$ and apply Lemma 2.2 to partition $H$ into subsets $H_{i}$, $1 \leq i \leq N_{l}$, such that

$$
\begin{aligned}
& \# H_{i} \geq \delta_{l} \# H \text { and } \\
& \text { the subsets } h_{1} H_{i}, \ldots, h_{l} H_{i} \text { are mutually disjoint }
\end{aligned}
$$

for every $i$. Denote by $r_{i}:\left(A^{l}\right)^{H} \rightarrow\left(A^{l}\right)^{H_{i}}$ the natural restriction map. Then we deduce from (2-3) that $r_{i} \circ \pi_{h_{1}, \cdots, h_{l}}$ maps $\lambda^{H}$ onto $\left(\lambda^{l}\right)^{H_{i}}$. Since $\operatorname{dist} \pi_{h_{1}, \ldots, h_{l}} x=$ $\sum_{i}\left(\# H_{i} / \# H\right) \cdot \operatorname{dist}\left(r_{i} \circ \pi_{h_{1}, \ldots, h_{l}}\right) x$, it follows that

$$
\begin{aligned}
\lambda^{H}\left(\left\{x \in A^{H} \mid \| \operatorname{dist}\right.\right. & \pi_{\left.\left.h_{1}, \ldots, h_{l} x-\lambda^{l} \|>\epsilon\right\}\right)} \\
& \leq \sum_{i} \lambda^{H}\left(\left\{x \in A^{H} \mid\left\|\operatorname{dist}\left(r_{i} \circ \pi_{h_{1}, \ldots, h_{l}}\right) x-\lambda^{l}\right\|>\epsilon\right\}\right) \\
& =\sum_{i}\left(\lambda^{l}\right)^{H_{i}}\left(\left\{y \in\left(A^{l}\right)^{H_{i}} \mid\left\|\operatorname{dist} y-\lambda^{l}\right\|>\epsilon\right\}\right) .
\end{aligned}
$$

By Lemma 2.2 and (2-2), there exists $c>0$ such that if $\# H$ is large enough then the $i$-th term in the latter sum is less then $e^{-c \# H_{i}}<e^{-c \delta_{l} \# H}$. Hence

$$
\lambda^{H}\left(B_{H}\right) \leq N_{l}\left(\begin{array}{c}
\# H \\
l
\end{array}\right) e^{-c \delta_{l} \# H}
$$

and the assertion of the lemma follows.

Now we are ready to define the sequence $\left(k_{n}, s_{n-1}\right)_{n \geq 1}$. Fix a sequence of positive reals $\epsilon_{n} \rightarrow 0$. On the first step one can take arbitrary $k_{1}$ and $s_{0}$. Suppose now-on the $n$-th step - we already have $k_{n}$ and $s_{n-1}$ and we want to define $k_{n+1}$ and $s_{n}$. For this, we apply Lemma 2.3 with $A:=F_{n}, l:=n$ and $\epsilon:=\epsilon_{n}$ to find $k_{n+1}$ large so that there exists $s_{n} \in A^{H_{n}}$ satisfying

$$
\left\|\operatorname{dist} \pi_{h_{1}, \ldots, h_{n}} s_{n}-\left(\lambda_{F_{n}}\right)^{n}\right\|<\epsilon_{n} \text { for all } h_{1} \neq \cdots \neq h_{n} \in H_{n} \text {. }
$$

Recall that $H_{n}:=\sum_{i=k_{n}+1}^{k_{n+1}} G_{i}$ and $F_{n}:=\sum_{i=1}^{k_{n}} G_{i}$ for $n \geq 1$. Without loss of generality we may also assume that $k_{n+1}-k_{n} \geq n$ and hence $\sum_{n=1}^{\infty}\left(\# H_{n}\right)^{-1}<\infty$.

Denote by $T$ the $(C, F)$-action of $G$ on $(X, \mathfrak{B}, \mu)$ associated with $\left(k_{n}, s_{n-1}\right)_{n=1}^{\infty}$.

Theorem 2.4. $T$ is mixing of any order.

Proof. (I) We first show that $T$ is mixing (of order 1 ).

Recall that a sequence $g_{n} \rightarrow \infty$ in $G$ is called mixing for $T$ if

$$
\lim _{n \rightarrow \infty} \mu\left(T_{g_{n}} B_{1} \cap B_{2}\right)=\mu\left(B_{1}\right) \mu\left(B_{2}\right) \text { for all } B_{1}, B_{2} \in \mathfrak{B}
$$

Clearly, $T$ is mixing if and only if any sequence going to infinity in $G$ contains a mixing subsequence. Since every subsequence of a mixing sequence is mixing itself, to prove (I) it suffices to show that every sequence $\left(g_{n}\right)_{n=1}^{\infty}$ in $G$ with $g_{n} \in F_{n+1} \backslash F_{n}$ 
for all $n$ is mixing. Notice first that there exist (unique) $f_{n} \in F_{n}$ and $h_{n} \in H_{n} \backslash\{1\}$ with $g_{n}=f_{n} \phi_{n}\left(h_{n}\right)$. Fix any two subsets $A, B \subset F_{n}$. We notice that for each $h \in H_{n}$,

$$
g_{n} A c_{n+1}(h)=f_{n} A s_{n}(h) \phi_{n}\left(h_{n} h\right)=f_{n} A s_{n}(h) s_{n}\left(h_{n} h\right)^{-1} c_{n+1}\left(h_{n} h\right)
$$

and $f_{n} A s_{n}(h) s_{n}\left(h_{n} h\right)^{-1} \subset F_{n}$. Hence

$$
\begin{aligned}
\mu\left(T_{g_{n}}[A]_{n} \cap[B]_{n}\right) & =\sum_{h \in H_{n}} \mu\left(T_{g_{n}}\left[A c_{n+1}(h)\right]_{n+1} \cap[B]_{n}\right) \\
& =\sum_{h \in H_{n}} \mu\left(\left[f_{n} A s_{n}(h) s_{n}\left(h_{n} h\right)^{-1} c_{n+1}\left(h_{n} h\right)\right]_{n+1} \cap[B]_{n}\right) \\
& =\sum_{h \in H_{n}} \mu\left(\left[\left(f_{n} A s_{n}(h) s_{n}\left(h_{n} h\right)^{-1} \cap B\right) c_{n+1}\left(h_{n} h\right)\right]_{n+1}\right) \\
& =\frac{1}{\# H_{n}} \sum_{h \in H_{n}} \mu\left(\left[f_{n} A s_{n}(h) s_{n}\left(h_{n} h\right)^{-1} \cap B\right]_{n}\right) \\
& =\frac{1}{\# H_{n}} \sum_{h \in H_{n}} \lambda_{F_{n}}\left(f_{n} A s_{n}(h) \cap B s_{n}\left(h_{n} h\right)\right) .
\end{aligned}
$$

We define a map $r_{A, B}: F_{n} \times F_{n} \rightarrow \mathbb{R}$ by setting

$$
r_{A, B}\left(g, g^{\prime}\right):=\lambda_{F_{n}}\left(f_{n} A g \cap B g^{\prime}\right) .
$$

Then it follows from (2-5) and (2-4) that

$$
\begin{aligned}
\mu\left(T_{g_{n}}[A]_{n} \cap[B]_{n}\right) & =\int_{F_{n} \times F_{n}} r_{A, B} d\left(\operatorname{dist} \pi_{1, h_{n}} s_{n}\right) \\
& =\int_{F_{n} \times F_{n}} r_{A, B} d \lambda_{F_{n} \times F_{n}} \pm \epsilon_{n} \\
& =\int_{F_{n} \times F_{n}} \lambda_{F_{n}}\left(f_{n} A g \cap B g^{\prime}\right) d \lambda_{F_{n}}(g) d \lambda_{F_{n}}\left(g^{\prime}\right) \pm \epsilon_{n} \\
& =\lambda_{F_{n}}(A) \lambda_{F_{n}}(B) \pm \epsilon_{n} \\
& =\mu\left([A]_{n}\right) \mu\left([B]_{n}\right) \pm \epsilon_{n} .
\end{aligned}
$$

Hence we have

$$
\max _{A, B \subset F_{n}}\left|\mu\left(T_{g_{n}}[A]_{n} \cap[B]_{n}\right)-\mu\left([A]_{n}\right) \mu\left([B]_{n}\right)\right|<\epsilon_{n} .
$$

This and (1-6) imply that the sequence $\left(g_{n}\right)_{n=1}^{\infty}$ is mixing.

(II) Now we fix $l>1$ and prove that $T$ is mixing of order $l$. To this end it is sufficient to show the following: given $l+1$ sequences $\left(g_{0, n}\right)_{n=1}^{\infty}, \ldots,\left(g_{l, n}\right)_{n=1}^{\infty}$ in $G$ such that $g_{i, n} \in F_{n+1}$ and $g_{i, n} g_{j, n}^{-1} \notin F_{n}$ whenever $i \neq j$,

$$
\max _{A_{0}, \ldots, A_{l}}\left|\mu\left(T_{g_{0, n}}\left[A_{0}\right]_{n} \cap \cdots \cap T_{g_{l, n}}\left[A_{l}\right]_{n}\right)-\mu\left(\left[A_{0}\right]_{n}\right) \cdots \mu\left(\left[A_{l}\right]_{n}\right)\right|<\epsilon_{n}
$$


for all $n>l$. Notice that for every $n \in \mathbb{N}$ and $0 \leq j \leq l$, there exist unique $f_{j, n} \in F_{n}$ and $h_{j, n} \in H_{n}$ with $g_{j, n}=f_{j, n} \phi_{n}\left(h_{j, n}\right)$. Moreover, $h_{0, n} \neq h_{2, n} \cdots \neq h_{1, n}$. Then slightly modifying the argument in (I), we compute

$$
\begin{aligned}
\mu\left(T_{g_{0, n}}\left[A_{0}\right]_{n}\right. & \left.\cap \cdots \cap T_{g_{l, n}}\left[A_{l}\right]_{n}\right) \\
= & \int_{F_{n}^{l}} \lambda_{F_{n}}\left(f_{0, n} A_{0} g_{0} \cap \cdots \cap f_{l, n} A_{l} g_{l}\right) d\left(\lambda_{F_{n}}\right)^{l+1}\left(g_{0}, \ldots, g_{l}\right) \pm \epsilon_{n} \\
= & \lambda_{F_{n}}\left(A_{0}\right) \cdots \lambda_{F_{n}}\left(A_{l}\right) \pm \epsilon_{n}=\mu\left(\left[A_{0}\right]_{n}\right) \cdots \mu\left(\left[A_{l}\right]_{n}\right) \pm \epsilon_{n} .
\end{aligned}
$$

To construct a weakly mixing rigid action of $G$ we define another sequence $\left(\widetilde{k}_{n}, \widetilde{s}_{n-1}\right)_{n \geq 1}$. When $n$ is odd, we choose $\widetilde{k}_{n}$ and $\widetilde{s}_{n-1}$ to satisfy the following weaker version of (2-4):

$$
\max _{1 \neq h \in H_{n}}\left\|\operatorname{dist} \pi_{1, h} s_{n}-\lambda_{F_{n}} \times \lambda_{F_{n}}\right\|<\epsilon_{n} .
$$

When $n$ is even, we just set $\widetilde{k}_{n}:=\widetilde{k}_{n-1}+1$ and $\widetilde{s}_{n} \equiv 1_{G}$. Denote by $\widetilde{T}$ the $(C, F)$-action of $G$ on $(\widetilde{X}, \widetilde{\mathfrak{B}}, \widetilde{\mu})$ associated with $\left(\widetilde{k}_{n}, \widetilde{s}_{n-1}\right)_{n=1}^{\infty}$.

Theorem 2.5. $\widetilde{T}$ is weakly mixing and rigid.

Proof. Take any sequence $h_{n} \in H_{2 n} \backslash\{1\}$. It follows from the part (I) of the proof of Theorem 2.4 and (2-8) that the sequence $\left(\phi_{2 n}\left(h_{n}\right)\right)_{n=1}^{\infty}$ is mixing for $\widetilde{T}$. Clearly, it is also mixing for $\widetilde{T} \times \widetilde{T}$. Hence $\widetilde{T} \times \widetilde{T}$ is ergodic, i.e. $\widetilde{T}$ is weakly mixing.

Now take any sequence $h_{n} \in H_{2 n+1} \backslash\{1\}$. Notice that (2-5) holds for any choice of $\left(k_{n}, s_{n-1}\right)_{n \geq 1}$. Hence we deduce from $(2-5)$ and the definition of $\widetilde{s}_{2 n+1}$ that

$$
\mu\left(\widetilde{T}_{\phi_{2 n+1}\left(h_{n}\right)}[A]_{2 n+1} \cap[B]_{2 n+1}\right)=\lambda_{F_{2 n+1}}(A \cap B)=\mu\left([A \cap B]_{2 n+1}\right)
$$

for all subsets $A, B \subset F_{2 n+1}$. This plus (1-6) yield

$$
\lim _{n \rightarrow \infty} \mu\left(\widetilde{T}_{\phi_{2 n+1}\left(h_{n}\right)} \widetilde{A} \cap \widetilde{B}\right)=\mu(\widetilde{A} \cap \widetilde{B})
$$

for all $\widetilde{A}, \widetilde{B} \in \widetilde{\mathfrak{B}}$. This means that $\widetilde{T}$ is rigid.

\section{Self-Joinings of $T$}

This section is devoted entirely to the proof of the following theorem.

Theorem 3.1. The action $T$ constructed in the previous section has MSJ.

Proof. (I) We first show that $T$ has $\mathrm{MSJ}_{2}$. Since $T$ is weakly mixing, we need to establish that

$$
J_{2}^{e}(T)=\left\{\mu_{g} \bullet \mid g \in G\right\} \cup\{\mu \times \mu\} .
$$

Take any $\nu \in J_{2}^{e}(T)$. Let $\mathfrak{F}_{n}$ denote the sub- $\sigma$-algebra of $\left(T_{g} \times T_{g}\right)_{g \in F_{n}}$-invariant subsets. Then $\mathfrak{F}_{1} \supset \mathfrak{F}_{2} \supset \cdots$ and $\bigcap_{n} \mathfrak{F}_{n}=\{\emptyset, X \times X\}(\bmod \nu)$. Since there are only countably many cylinders, we deduce from the martingale convergence theorem that for $\nu$-a.a. $\left(x, x^{\prime}\right)$,

$$
E\left(\chi_{B \times B^{\prime}} \mid \mathfrak{F}_{n-1}\right)\left(x, x^{\prime}\right)=\frac{1}{\# F_{n-1}} \sum_{\substack{g \in F_{n-1} \\ 9}} \chi_{B \times B^{\prime}}\left(T_{g} x, T_{g} x^{\prime}\right) \rightarrow \nu\left(B \times B^{\prime}\right)
$$


as $n \rightarrow \infty$ for any pair of cylinders $B, B^{\prime} \subset X$. Fix such a point $\left(x, x^{\prime}\right)$. It is called generic for $(T \times T, \nu)$. Given any $n>0$, we can write $x$ and $x^{\prime}$ as infinite sequences

$$
x=\left(f_{n}, d_{n+1}, d_{n+2}, \ldots\right) \text { and } x^{\prime}=\left(f_{n}^{\prime}, d_{n+1}^{\prime}, d_{n+2}^{\prime}, \ldots\right)
$$

with $f_{n}, f_{n}^{\prime} \in F_{n}$ and $d_{i}, d_{i}^{\prime} \in C_{i}$ for all $i>n$. Recall that $f_{n}:=f_{0} d_{1} \cdots d_{n}$ and $f_{n}^{\prime}:=f_{0}^{\prime} d_{1}^{\prime} \cdots d_{n}^{\prime}$. We set $t_{n}:=f_{n}^{\prime} f_{n}^{-1}, n>0$. Fix a pair of cylinders, say $m$ cylinders, $B$ and $B^{\prime}$. If $n>m$ and $g \in F_{n}$ then $T_{g} x^{\prime}=\left(g f_{n}^{\prime}, d_{n+1}^{\prime}, d_{n+2}^{\prime}, \ldots\right)$. Hence $T_{g} x^{\prime} \in B^{\prime}$ if and only if $T_{g} T_{t_{n}} x \in B^{\prime}$. Therefore

$$
\chi_{B \times B^{\prime}}\left(T_{g} x, T_{g} x^{\prime}\right)=\chi_{T_{g}^{-1} B \cap T_{t_{n}}^{-1} T_{g}^{-1} B^{\prime}}(x) .
$$

Since $x$ is generic for $(T, \mu)$, it follows that

$$
\lim _{l \rightarrow \infty} \frac{1}{\# F_{l}} \sum_{a \in F_{l}} \chi_{T_{g}^{-1} B \cap T_{t_{n}}^{-1} T_{g}^{-1} B^{\prime}}\left(T_{a} x\right)=\mu\left(T_{g}^{-1} B \cap T_{t_{n}}^{-1} T_{g}^{-1} B^{\prime}\right) .
$$

Therefore (3-1) yields

$$
\lim _{n \rightarrow \infty} \frac{1}{\# F_{n-1}} \sum_{g \in F_{n-1}} \mu\left(T_{g}^{-1} B \cap T_{t_{n}}^{-1} T_{g}^{-1} B^{\prime}\right)=\nu\left(B \times B^{\prime}\right) .
$$

Consider now two cases. If $t_{n} \notin F_{n-1}$ for infinitely many $n$ then passing to the limit in (3-2) along this subsequence and making use of (2-6) we obtain that $\mu(B) \mu\left(B^{\prime}\right)=$ $\nu\left(B \times B^{\prime}\right)$. Hence $\mu \times \mu=\nu$. If, otherwise, there exists $N>0$ such that $t_{n} \in F_{n-1}$, i.e. $d_{n}=d_{n}^{\prime}$, for all $n>N$ then $x$ and $x^{\prime}$ are $T$-orbit equivalent, $t_{n}=t_{N}$ and

$$
\begin{aligned}
\frac{1}{\# F_{n-1}} \sum_{g \in F_{n-1}} \mu\left(T_{g}^{-1} B \cap T_{t_{n}}^{-1} T_{g}^{-1} B^{\prime}\right) & =\frac{1}{\# F_{N}} \sum_{g \in F_{N}} \mu\left(B \cap T_{g} T_{t_{N}}^{-1} T_{g}^{-1} B^{\prime}\right) \\
& =\mu_{\left(t_{N}^{-1}\right)}\left(B \times B^{\prime}\right)
\end{aligned}
$$

Passing to the limit in (3-1) we obtain that $\nu=\mu_{\left(t_{N}^{-1}\right)} \bullet$

(II) Now we fix $l>1$ and show that $T$ has $\mathrm{MSJ}_{l+1}$. Take any joining $\nu \in J_{l+1}^{e}(T)$ and fix a generic point $\left(x_{0}, \ldots, x_{l}\right)$ for $(T \times \cdots \times T, \nu)$. Define a partition $P$ of $\{0, \ldots, l\}$ by setting: $i_{1}$ and $i_{2}$ are in the same atom of $P$ if $x_{i_{1}}$ and $x_{i_{2}}$ are $T$-orbit equivalent. As in (I), for any $n$, we can write

$$
x_{j}=\left(f_{j, n-1}, d_{j, n}, d_{j, n+1}, \ldots\right) \in X_{n-1}, \quad j=0, \ldots, l .
$$

Suppose first that $\# P=l+1$, i.e. $P$ is the finest possible. Then by the proof of (I), each 2-dimensional marginal of $\nu$ is $\mu \times \mu$. Since $\sum_{i=1}^{\infty}\left(\# C_{i}\right)^{-1}<\infty$ and $\mu=\lambda_{F_{0}} \times \lambda_{C_{1}} \times \lambda_{C_{2}} \times \cdots$, it follows from the Borel-Cantelli lemma that for $\nu$-a.a. $\left(y_{0}, \ldots, y_{l}\right) \in X^{l+1}$

$$
\exists N>0 \text { such that } y_{0, i} \neq y_{1, i} \neq \cdots \neq y_{l, i} \text { whenever } i>N,
$$

where $y_{j, i} \in C_{i}$ is the $i$-th coordinate of $y_{j} \in F_{0} \times C_{1} \times C_{2} \times \cdots$. Hence without loss of generality we may assume that this condition is satisfied for $\left(x_{0}, \ldots, x_{l}\right)$. Thus, if we set $t_{j, n}:=f_{j, n} f_{0, n}{ }^{-1}=f_{j, n-1} d_{j, n} d_{0, n}{ }^{-1} f_{0, n-1}{ }^{-1}$ then $t_{j, n} t_{i, n}^{-1} \notin F_{n-1}$ 
whenever $i \neq j$. Slightly modifying our reasoning in (I) and making use of (2-7) instead of (2-6) we now obtain

$$
\begin{aligned}
\nu\left(B_{0} \times \cdots \times B_{l}\right) & =\lim _{n \rightarrow \infty} \sum_{g \in F_{n-1}} \chi_{B_{0} \times \cdots \times B_{l}}\left(T_{g} x_{0}, \ldots T_{g} x_{l}\right) \\
& =\lim _{n \rightarrow \infty} \sum_{g \in F_{n-1}} \chi_{B_{0} \times \cdots \times B_{l}}\left(T_{g} x_{0}, T_{g} T_{t_{1, n}} x_{0}, \ldots, T_{g} T_{t_{l, n}} x_{0}\right) \\
& =\lim _{n \rightarrow \infty} \sum_{g \in F_{n-1}} \mu\left(T_{g} B_{0} \cap T_{t_{1, n}}^{-1} T_{g} B_{1} \cap \cdots \cap T_{t_{l, n}}^{-1} T_{g} B_{l}\right) \\
& =\mu\left(B_{0}\right) \cdots \mu\left(B_{l}\right)
\end{aligned}
$$

for any $(l+1)$-tuple of cylinders $B_{0}, \ldots, B_{l}$. Hence $\nu=\mu \times \cdots \times \mu$.

Consider now the general case and put $t_{j, n}:=f_{j, n} f_{i_{p}, n}^{-1}$ for each $j \in p, p \in P$. Recall that $i_{p}=\min _{j \in p} j$. Then

$$
\chi_{B_{0} \times \cdots \times B_{l}}\left(T_{g} x_{0}, \ldots, T_{g} x_{l}\right)=\prod_{p \in P} \chi_{A_{p}}\left(x_{i_{p}}\right),
$$

where $A_{p}:=\bigcap_{j \in p} T_{t_{j, n}}^{-1} T_{g}^{-1} B_{j}$. Notice that the point $\left(x_{i_{p}}\right)_{p \in P} \in X^{\left\{i_{p} \mid p \in P\right\}}$ is generic for $(T \times \cdots \times T$ (\#P times), $\kappa)$, where $\kappa$ stands for the projection of $\nu$ onto $X^{\left\{i_{p} \mid p \in P\right\}}$. By the first part of (II), $\kappa=\mu \times \cdots \times \mu$ (\#P times). Hence

$$
\begin{aligned}
\nu\left(B_{0} \times \cdots \times B_{l}\right) & =\lim _{n \rightarrow \infty} \frac{1}{\# F_{n-1}} \sum_{g \in F_{n-1}} \chi_{B_{0} \times \cdots \times B_{l}}\left(T_{g} x_{0}, \ldots T_{g} x_{l}\right) \\
& =\lim _{n \rightarrow \infty} \frac{1}{\# F_{n-1}} \sum_{g \in F_{n-1}} \prod_{p \in P} \mu\left(A_{p}\right) .
\end{aligned}
$$

As in (I), a 'stabilization' property holds: there exists $M>0$ such that $t_{j, n}=t_{j, M}$ for all $n>M$. We now set $g:=\left(t_{0, M}^{-1}, \ldots, t_{l, M}^{-1}\right)$. Clearly, $g$ is $P$-subordinated. Hence

$$
\nu\left(B_{0} \times \cdots \times B_{l}\right)=\frac{1}{\# F_{M}} \sum_{g \in F_{M}} \prod_{p \in P} \mu\left(\bigcap_{j \in p} T_{g} T_{t_{j, M}} T_{g}^{-1} B_{j}\right)=\mu_{g} \bullet\left(B_{0} \times \cdots \times B_{l}\right) .
$$

\section{UNCOUNTABLY MANY MIXING ACTIONS WITH MSJ}

In this section the proof of Theorems $0.1(\mathrm{i})$ and 0.4 will be completed. We first apply Lemma 2.3 to construct $k_{n+1}$ and $s_{n}, \widehat{s}_{n} \in F_{n}^{H_{n}}$ in such a way that (2-4) is satisfied for both $s_{n}$ and $\widehat{s}_{n}$ and, in addition,

$$
\left\|\operatorname{dist}_{h \in H_{n}}\left(s_{n}(h k), \widehat{s}_{n}\left(h k^{\prime}\right)\right)-\lambda_{F_{n}} \times \lambda_{F_{n}}\right\|<\epsilon_{n}
$$

for all $k, k^{\prime} \in H_{n}$. Next, given $\sigma \in\{0,1\}^{\mathbb{N}}$ and $n \in \mathbb{N}$, we define $s_{n}^{\sigma}: H_{n} \rightarrow F_{n}$ by setting

$$
s_{n}^{\sigma}=\left\{\begin{array}{l}
s_{n} \text { if } \sigma(n)=0, \\
\widehat{s}_{n} \text { if } \sigma(n)=1 .
\end{array}\right.
$$

Now we denote by $T^{\sigma}$ the $(C, F)$-action of $G$ associated with $\left(k_{n}, s_{n-1}^{\sigma}\right)_{n=1}^{\infty}$. Let $\Sigma$ be an uncountable subset of $\{0,1\}^{\mathbb{N}}$ such that for any $\sigma, \sigma^{\prime} \in \Sigma$, the subset $\left\{n \in \mathbb{N} \mid \sigma(n) \neq \sigma^{\prime}(n)\right\}$ is infinite. 


\section{Theorem 4.1.}

(i) For any $\sigma \in\{0,1\}^{\mathbb{N}}$, the action $T^{\sigma}$ is mixing and has MSJ.

(ii) If $\sigma, \sigma^{\prime} \in \Sigma$ and $\sigma \neq \sigma^{\prime}$ then $T^{\sigma}$ and $T^{\sigma^{\prime}}$ are disjoint.

Proof. (i) follows from the proof of Theorem 3.1, since (2-4) is satisfied for $s_{n}^{\sigma}$ for all $\sigma \in\{0,1\}^{\mathbb{N}}$ and $n \in \mathbb{N}$.

(ii) Let $\nu \in J^{e}\left(T^{\sigma}, T^{\sigma^{\prime}}\right)$. Take a generic point $\left(x, x^{\prime}\right)$ for $\left(T^{\sigma} \times T^{\sigma^{\prime}}, \nu\right)$. Consider any $n$ such that $\sigma(n) \neq \sigma^{\prime}(n)$. Then we can write $x$ and $x^{\prime}$ as infinite sequences $x=$ $\left(f_{n}, d_{n+1}, d_{n+2}, \ldots\right)$ and $x^{\prime}=\left(f_{n}^{\prime}, d_{n+1}^{\prime}, d_{n+2}^{\prime}, \ldots\right)$ with $f_{n}, f_{n}^{\prime} \in F_{n}$ and $d_{m}, d_{m}^{\prime} \in$ $C_{m}$ for all $m>n$. Take any $g \in F_{n+1}$. Then we have the following expansions

$$
g=a \phi_{n}(h), d_{n+1}=s_{n}^{\sigma}\left(h_{n}\right) \phi_{n}\left(h_{n}\right) \text { and } d_{n+1}^{\prime}=s_{n}^{\sigma^{\prime}}\left(h_{n}^{\prime}\right) \phi_{n}\left(h_{n}^{\prime}\right)
$$

for some uniquely determined $a \in F_{n}$ and $h, h_{n}, h_{n}^{\prime} \in H_{n}$. Since

$$
\begin{aligned}
& g f_{n} d_{n+1}=a f_{n} s_{n}^{\sigma}\left(h_{n}\right) s_{n}^{\sigma}\left(h h_{n}\right)^{-1} c_{n+1}\left(h h_{n}\right) \text { and } \\
& g f_{n}^{\prime} d_{n+1}^{\prime}=a f_{n}^{\prime} s_{n}^{\sigma^{\prime}}\left(h_{n}^{\prime}\right) s_{n}^{\sigma^{\prime}}\left(h h_{n}^{\prime}\right)^{-1} c_{n+1}\left(h h_{n}^{\prime}\right),
\end{aligned}
$$

the following holds for any pair of subsets $A, A^{\prime} \subset F_{n}$ :

$$
\begin{aligned}
& \frac{\#\left\{g \in F_{n+1} \mid\left(T_{g}^{\sigma} x, T_{g}^{\sigma^{\prime}} x^{\prime}\right) \in[A]_{n} \times\left[A^{\prime}\right]_{n}\right\}}{\# F_{n+1}} \\
& =\frac{1}{\# F_{n}} \sum_{a \in F_{n}} \frac{\#\left\{h \in H_{n} \mid a f_{n} s_{n}^{\sigma}\left(h_{n}\right) s_{n}^{\sigma}\left(h h_{n}\right)^{-1} \in A, a f_{n}^{\prime} s_{n}^{\sigma^{\prime}}\left(h_{n}^{\prime}\right) s_{n}^{\sigma^{\prime}}\left(h h_{n}^{\prime}\right)^{-1} \in A^{\prime}\right\}}{\# H_{n}} \\
& =\frac{1}{\# F_{n}} \sum_{a \in F_{n}} \xi_{n}\left(A^{-1} a f_{n} s_{n}^{\sigma}\left(h_{n}\right) \times A^{\prime-1} a f_{n}^{\prime} s_{n}^{\sigma^{\prime}}\left(h_{n}^{\prime}\right)\right),
\end{aligned}
$$

where $\xi_{n}:=\operatorname{dist}_{h \in H_{n}}\left(s_{n}^{\sigma}\left(h h_{n}\right), s_{n}^{\sigma^{\prime}}\left(h h_{n}^{\prime}\right)\right)$. This and (4-1) yield

$$
\begin{aligned}
\frac{\#\left\{g \in F_{n+1} \mid\left(T_{g}^{\sigma} x, T_{g}^{\sigma^{\prime}} x^{\prime}\right) \in[A]_{n} \times\left[A^{\prime}\right]_{n}\right\}}{\# F_{n+1}} & =\lambda_{F_{n}}(A) \lambda_{F_{n}}\left(A^{\prime}\right) \pm \epsilon_{n} \\
& =\mu\left([A]_{n}\right) \mu\left(\left[A^{\prime}\right]_{n}\right) \pm \epsilon_{n} .
\end{aligned}
$$

Since $\left(x, x^{\prime}\right)$ is generic for $\left(T^{\sigma} \times T^{\sigma^{\prime}}, \nu\right)$ and (4-2) holds for infinitely many $n$, we deduce that $\nu=\mu \times \mu$.

By refining the above argument the reader can strengthen Theorem 0.1(i) as follows: there exists an uncountable family of mixing (of any order) rank-one $G$ actions with MSJ such that any finite subfamily of it is disjoint.

\section{ON G-ACTIONS With MSJ}

It follows immediately from Definition 0.2 that if $T$ has $\mathrm{MSJ}_{2}$ then the centralizer $C(T)$ of $T$ is 'trivial', i.e. $C(T)=\left\{T_{g} \mid g \in C(G)\right\}$, where $C(G)$ denotes the center of $G$. Moreover, we will show that $T$ has trivial product centralizer (as D. Rudolph $\operatorname{did}$ in $[\mathrm{Ru}]$ for $\mathbb{Z}$-actions).

Let $\left(X^{l}, \mathfrak{B}^{\otimes l}, \mu^{l}, T^{(l)}\right)$ denote the $l$-fold Cartesian product of $(X, \mathfrak{B}, \mu, T)$. Given a permutation $\sigma$ of $\{1, \ldots, l\}$ and $g_{1}, \ldots, g_{n} \in C(T)$, we define a transformation $U_{\sigma, g_{1}, \ldots, g_{l}}$ of $\left(X^{l}, \mathfrak{B}^{\otimes l}, \mu^{l}, T^{(l)}\right)$ by setting

$$
U_{\sigma, g_{1}, \ldots, g_{l}}\left(x_{1}, \ldots, x_{l}\right):=\left(T_{g_{1}} x_{\sigma(1)}, \ldots, T_{g_{l}} x_{\sigma(l)}\right) .
$$

Of course, $U_{\sigma, g_{1}, \ldots, g_{l}} \in C\left(T^{(l)}\right)$. We show that for the actions with MSJ, the converse also holds. 
Proposition 5.1. If $T$ has $M S J$ then for any $l \in \mathbb{N}$, each element of $C\left(T^{(l)}\right)$ equals to $U_{\sigma, g_{1}, \ldots, g_{l}}$ for some permutation $\sigma$ and elements $g_{1}, \ldots, g_{l} \in C(G)$.

Proof. Let $S \in C\left(T^{(l)}\right)$. We define an ergodic 2-fold self-joining $\nu$ of $T^{(l)}$ by setting $\nu(A \times B):=\mu^{l}\left(A \cap S^{-1} B\right)$ for all $A, B \in \mathfrak{B}^{\otimes l}$. Notice that $\nu \in J_{2 l}^{e}(T)$. Since $T$ has $\mathrm{MSJ}_{2 l}$, there exists a partition $P$ of $\{1, \ldots, 2 l\}$ and a $P$-subordinated element $g=\left(g_{1}, \ldots, g_{2 l}\right) \in \mathrm{FC}(G)^{2 l}$ such that

$$
\nu\left(A_{1} \times \cdots \times A_{2 l}\right)=\frac{1}{\# g^{\bullet 2 l}} \sum_{\left(h_{1}, \ldots, h_{2 l}\right) \in g^{\bullet 2 l}} \prod_{p \in P} \mu\left(\bigcap_{i \in p} T_{h_{i}} A_{i}\right) .
$$

for all subsets $A_{1}, \ldots, A_{2 l} \in \mathfrak{B}$. Substituting at first $A_{1}=\cdots=A_{l}=X$ and then $A_{l+1}=\cdots=A_{2 l}=X$ in (5-1) we derive that $\# P=l$, \#p=2 for all $p \in P$ and $\# g^{\bullet 2 l}=1$. Hence $g_{1}, \ldots, g_{2 l} \in C(G)$ and there exists a bijection $\sigma$ of $\{1, \ldots, l\}$ such that $P=\{\{i, \sigma(i)+l\} \mid i=1, \ldots, l\}$. Therefore in follows from (5-1) that

$$
S^{-1}\left(A_{l+1} \times \cdots \times A_{2 l}\right)=T_{g_{l+1}} A_{l+\sigma(1)} \times \cdots \times T_{g_{2 l}} A_{l+\sigma(l)} .
$$

As a simple corollary we derive that if $T$ has MSJ then the $G$-actions $T, T^{(2)}, \ldots$ and $T \times T \times \cdots$ are pairwise non-isomorphic.

After this paper was submitted the author introduced a companion to MSJ concept of simplicity for actions of locally compact second countable groups [Da3]. As appeared, this concept is more general that the simplicity in the sense of A. del Junco and D. Rudolph $[\mathrm{JuR}]$ even for $\mathbb{Z}$-actions. For instance, there exist simple transformations which are disjoint from all 2-fold del Junco-Rudolph's-simple ones. It is shown in [Da3] that an analogue of Veech theorem on the structure of factors holds for this extended class of simple actions. In particular, if $T$ has $\mathrm{MSJ}_{2}$ then for every non-trivial factor $\mathfrak{F}$ of $T$ there exists a compact normal subgroup $K$ of $G$ such that

$$
\mathfrak{F}=\operatorname{Fix} K:=\left\{A \in \mathfrak{B} \mid \mu\left(T_{k} A \triangle A\right)=0 \text { for all } k \in K\right\} .
$$

Thus if $T$ has $\mathrm{MSJ}_{2}$ then $T$ is effectively prime, i.e. $T$ has no effective factors. (Recall that a $G$-action $Q$ is called effective if $Q_{g} \neq \operatorname{Id}$ for each $g \neq 1_{G}$.)

\section{REFERENCES}

[Ad] T. Adams, Smorodinsky's conjecture on rank one systems, Proc. Amer. Math. Soc. 126 (1998), 739-744.

[AdS] T. Adams and C. E. Silva, $\mathbb{Z}^{d}$-staircase actions, Ergod. Th. \& Dynam. Sys. 19 (1999), 837-850.

[CrS] D. Creutz and C. E. Silva, Mixing on a class of rank-one transformations, Ergod. Th. \& Dynam. Sys. 24 (2004), 407-440.

[Da1] A. I. Danilenko, Funny rank-one weak mixing for nonsingular Abelian actions, Isr. J. Math. 121 (2001), 29-54.

[Da2] Infinite rank one actions and nonsingular Chacon transformations, Illinois J. Math. 48 (2004), 769-786.

[Da3] , On simplicity concepts for ergodic actions, in preparation.

[DaS1] A. I. Danilenko and C. E. Silva, Multiple and polynomial recurrence for Abelian actions in infinite measure, J. London Math. Soc. (2) 69 (2004), 183-200.

[DaS2] Mixing rank-one actions of locally compact Abelian groups, preprint.

[Fa] B. Fayad, Rank one and mixing differentiable flows, Invent. Math. 160 (2005), 305-340. 
[Ju] A. del Junco, A simple map with no prime factors, Isr. J. Math. 104 (1998), 301-320.

[JuR] A. del Junco and D. Rudolph, On ergodic actions whose self-joinings are graphs, Ergod. Th. \& Dynam. Sys. 7 (1987), 531-557.

[JuY] A. del Junco and R. Yassawi, Multiple mixing rank one group actions, Canadian J. Math. 52 (2000), 332-347.

[Ka] S. A. Kalikow, Twofold mixing implies threefold mixing for rank one transformations, Ergodic Theory Dynam. Systems 4 (1984), 237-259.

[Ki] J. L. King, Joining-rank and the structure of finite rank mixing transformations, J. d'Analyse Math. 51 (1988), 182-227.

[Ma] B. Madore, Rank-one group actions with simple mixing $\mathbb{Z}$-subactions, New York J. Math. 10 (2004), 175-194.

[Or] D. S. Ornstein, On the root problem in ergodic theory, Proc. Sixth Berkley Symp. Math. Stat. Prob. (Univ. California, Berkeley, Calif., 1970/1971), vol. II, Univ. of California Press, Berkeley, Calif., 1972, pp. 347-356.

[Pr] A. Prikhodko, Stochastic constructions of flows of rank one, Mat. Sb. 192 (2001), 61-92.

[Ru] D. J. Rudolph, An example of a measure preserving map with minimal self-joinings, J. d'Analyse Math. 35 (1979), 97-122.

[Ry] V. V. Ryzhikov, Mixing, rank and minimal self-joining of actions with invariant measure, Mat. Sb. 183 (1992), 133-160.

Institute for Low Temperature Physics \& Engineering of Ukrainian National Academy of Sciences, 47 Lenin Ave., Kharkov, 61164, UKRAINE

E-mail address: danilenko@ilt.kharkov.ua 